\title{
Soft Pair-Wise Continuity in Soft Bitopological Spaces
}

\author{
Taha Yasin Ozturk ${ }^{*}$, Cigdem Gunduz Aras ${ }^{2}$ \\ ${ }^{1}$ Kafkas University, Faculty of Science and Letter, Department of Mathematic, \\ 36100, Kars, Turkey, taha36100@hotmail.com \\ ${ }^{2}$ Kocaeli University, Faculty of Science and Letter, Department of Mathematic, \\ 41380, Kocaeli, Turkey, carasgunduz@gmail.com \\ ${ }^{*}$ Corresponding Author \\ Received: $12^{\text {th }}$ December 2016 \\ Accepted: $13^{\text {th }}$ March 2017 \\ DOI: $10.18466 /$ cbayarfbe.319906
}

\begin{abstract}
The purpose of this paper is to introduce the concept of soft pair-wise neighborhood system in soft bitopological spaces. The notions of soft pair-wise contunity, soft pair-wise open (closed) mappings and soft pair-wise homeomorphism on soft bitopological space are introduced and their basic properties are investigated.
\end{abstract}

Keywords - Soft set, Soft point, Soft topological space, Soft bitopological spaces, Soft pair-wise neighborhood, Soft pair-wise contunious mappings, Soft pair-wise homeomorphism.

\section{Introduction}

Many practical problems in economics, engineering, environment, social science, medical science etc. cannot be dealt with classical methods because classical methods have inherent difficulties. The reason for these difficulties may be due to the inadequacy of the theories of parameterization tools. Molodtsov [15] initiated the concept of soft set theory as a new mathematical tool for dealing with uncertainties. Since soft set theory has a rich potential, researchs [11], [12], [2] on soft set theory and its applications in various fields are progressing rapidly. Recently, Shabir and Naz [19] have initiated the study of soft topological spaces. Theoretical studies of soft topological spaces have also been researched by some authors in [1], [3], [4], [6], [7], [14], [16], [17], [20], [21].

The concept of bitopological spaces was introduced by Kelly [10] as an extension of topological spaces in 1963. In later years, many researchers studied bitopological spaces and pair-wise open (closed) sets. Subsequently, Mashhour A. S. et al., [13] introduced supratopolological spaces as another extension of topological spaces. Therefore, handling of these spaces in soft concept is important and actual (e.g. [5], [8], [9], [18]).

In section 2 of the present paper, some preliminary definitions and theorems about soft topological spaces, soft bitopological spaces and soft supratopological spaces are given. Section 3, we introduce the concept of soft pair-wise neighborhood system in soft bitopological spaces. The properties of these concept and some important results related to soft supratopology are obtained. Section 4, is given soft pair-wise contunity on soft bitopological spaces and its properties has investigated. Finally, soft pairwise open (closed) mappings and soft pair-wise homeomorphism are defined and some examples are derived.

\section{Preliminaries}

In this section we will introduce necessary definitions and theorems for soft sets. Molodtsov [15] defined the soft set in the following way:

Let $X$ be an initial universe set and $E$ be a set of 
parameters. Let $P(X)$ denotes the power set of $X$ and $A, B \subseteq E$.

Definition 2.1 [15] A pair $(F, A)$ is called a soft set over $X$, where $F$ is a mapping given by $F: A \rightarrow P(X)$.

In other words, the soft set is a parameterized family of subsets of the set $X$. For $e \in A, F(e)$ may be considered as the set of $e$-elements of the soft set $(F, A)$, or as the set of $e$-approximate elements of the soft set, i.e.,

$$
(F, A)=\{(e, F(e)): e \in A \subseteq E, F: A \rightarrow P(X)\} .
$$

Definition 2.2 [12] For two soft sets $(F, A)$ and $(G, B)$ over $X,(F, A)$ is called soft subset of $(G, B)$ if

1. $A \subset B$ and

2. $\forall e \in A, F(e)$ and $G(e)$ are identical approximations.

This relationship is denoted by $(F, A) \widetilde{\subseteq}(G, B)$. Similarly, $(F, A)$ is called a soft superset of $(G, B)$ if $(G, B)$ is a soft subset of $(F, A)$. This relationship is denoted by $(F, A) \cong(G, B)$. Two soft sets $(F, A)$ and $(G, B)$ over $X$ are called soft equal if $(F, A)$ is a soft subset of $(G, B)$ and $(G, B)$ is a soft subset of $(F, A)$.

Definition 2.3 [12] The intersection of two soft sets $(F, A)$ and $(G, B)$ over $X$ is the soft set $(H, C)$, where $C=A \cap B$ and $\forall e \in C, H(e)=F(e) \cap G(e)$. This is denoted by $(F, A) \widetilde{\cap}(G, B)=(H, C)$.

Definition 2.4 [12] The union of two soft sets $(F, A)$ and $(G, B)$ over $X$ is the soft set, where $C=A \cup B$ and $\forall e \in C$,

$$
H(\varepsilon)= \begin{cases}F(e), & \text { if } e \in A-B, \\ G(e), & \text { if } e \in B-A, \\ F(e) \cup G(e) & \text { if } e \in A \cup B .\end{cases}
$$

This relationship is denoted by $(F, A) \tilde{\cup}(G, B)=$ $(H, C)$.

Definition 2.5 [12] A soft set $(F, A)$ over $X$ is said to be a NULL soft set denoted by $\Phi$ if for all $e \in A$, $F(e)=\emptyset$ (null set).

Definition 2.6 [12] Asoft set $(F, A)$ over $X$ is said to be an absolute soft set denoted by $\tilde{X}$ if for all $e \in A$, $F(e)=X$.
Definition 2.7 [19] The difference $(H, E)$ of two soft sets $(F, E)$ and $(G, E)$ over $X$, denoted by $(F, E) \backslash$ $(G, E)$, is defined as $H(e)=F(e) \backslash G(e)$ for all $e \in E$.

Definition 2.8 [19] Let $Y$ be a non-empty subset of $X$, then $\tilde{Y}$ denotes the soft set $(Y, E)$ over $X$ for which $Y(e)=Y$, for all $e \in E$.

In particular, $(X, E)$ will be denoted by $\tilde{X}$.

Definition 2.9 [19] The complement of a soft set $(F, E)$, denoted by $(F, E)^{c}$, is defined $(F, E)^{c}=\left(F^{c}, E\right)$, where $F^{c}: E \rightarrow P(X)$ is a mapping given by $F^{c}(e)=$ $X \backslash F(e), \forall e \in E$ and $F^{c}$ is called the soft complement function of $F$.

Definition 2.10 [19] Let $\tau$ be the collection of soft set over $X$, then $\tau$ is said to be a soft topology on $X$ if

1. $\Phi, \tilde{X}$ belongs to $\tau$;

2. the union of any number of soft sets in $\tau$ belongs to $\tau$;

3. The intersection of any two soft sets in $\tau$ belongs to $\tau$.

The triplet $(X, \tau, E)$ is called a soft topological space over $X$.

Definition 2.11 [19] Let $(X, \tau, E)$ be a soft topological space over $X$, then members of $\tau$ are said to be soft open sets in $X$.

Definition 2.12 [19] Let $(X, \tau, E)$ be a soft topological space over $X$. A soft set $(F, E)$ over $X$ is said to be a soft closed in $X$, if its complement $(F, E)^{c}$ belongs to $\tau$.

Proposition 2.1 [19] Let $(X, \tau, E)$ be a soft topological space over $X$. Then the collection $\tau_{e}=\{F(e):(F, E) \in$ $\tau$ \} for each $e \in E$, defines a topology on $X$.

Definition 2.13 [19] Let $(X, \tau, E)$ be a soft topological space over $X$ and $(F, E)$ be a soft set over $X$. Then the soft closure of $(F, E)$, denoted by $c l_{\tau}^{S}(F, E)$ is the intersection of all soft closed super sets of $(F, E)$. Clearly $c l_{\tau}^{S}(F, E)$ is the smallest soft closed set over $X$ which contains $(F, E)$.

Definition 2.14 [3] Let $(F, E)$ be a soft set over $X$. The soft set $(F, E)$ is called a soft point, denoted by 
$\left(x_{e}, E\right)$, if for the element $e \in E, F(e)=\{x\}$ and $F\left(e^{c}\right)=\emptyset$ for all $e^{c} \in E-\{e\}$ (briefly denoted by $x_{e}$ ).

Definition 2.15 [3] Two soft points $\left(x_{e}, E\right)$ and $\left(y_{e^{\prime}}, E\right)$ over a common universe $X$, we say that the points are different points if $x \neq y$ or $e \neq e^{\prime}$.

Definition 2.16 [3] The soft point $x_{e}$ is said to be belonging to the soft set $(F, E)$, denoted by $x_{e} \widetilde{\in}(F, E)$, if $x_{e}(e) \in F(e)$,i.e., $\{x\} \subseteq F(e)$.

Definition 2.17 [3] Let $(X, \tau, E)$ be a soft topological space over $X$. A soft set $(F, E)$ in $(X, \tau, E)$ is called a soft neighborhood of the soft point $\left(x_{e}, E\right) \widetilde{\in}(F, E)$ if there exists a soft open set $(G, E)$ such that $\left(x_{e}, E\right) \widetilde{\in}(G, E) \widetilde{\subset}(F, E)$.

Definition 2.18 [6] Let $(X, \tau, E)$ and $\left(Y, \tau^{\prime}, E\right)$ be two soft topological spaces, $f:(X, \tau, E) \rightarrow\left(Y, \tau^{\prime}, E\right)$ be a mapping. For each soft neighbourhood $(H, E)$ of $\left(f(x)_{e}, E\right)$, if there exists a soft neighbourhood $(F, E)$ of $\left(x_{e}, E\right)$ such that $f((F, E)) \widetilde{\subset}(H, E)$, then $f$ is said to be soft continuous mapping at $\left(x_{e}, E\right)$.

If $f$ is soft continuous mapping for all $\left(x_{e}, E\right)$

Definition 2.19 [6] Let $(X, \tau, E)$ and $\left(Y, \tau^{\prime}, E\right)$ be two soft topological spaces, $f: X \rightarrow Y$ be a mapping. If $f$ is a bijection, soft continuous and $f^{-1}$ is a soft continuous mapping, then $f$ is said to be soft homeomorphism from $X$ to $Y$. When a homeomorphism $f$ exists between $X$ and $Y$, we say that $X$ is soft homeomorphic to $Y$.

Definition 2.20 [1] Let $\left\{\left(\varphi_{s}, \psi_{s}\right):(X, \tau, E) \rightarrow\right.$ $\left.\left(Y_{S}, \tau_{S}, E_{S}\right)\right\}_{s \in S}$ be a family of soft mappings and $\left\{\left(Y_{S}, \tau_{S}, E_{S}\right)\right\}_{s \in S}$ is a family of soft topological spaces. Then, the topology $\tau$ generated from the subbase $\delta=$ $\left\{\left(\varphi_{s}, \psi_{s}\right)_{s \in S}^{-1}(F, E):(F, E) \in \tau_{s}, s \in S\right\}$ is called the soft topology (or initial soft topology) induced by the family of soft mappings $\left\{\left(\varphi_{s}, \psi_{s}\right)\right\}_{s \in S}$.

Definition 2.21 [5] Let $\mu$ be a collection of soft sets over $X$. Then, $\mu$ is said to be a soft supratopology on $X$ if it satisfies the following conditions:

1. $(\tilde{X}, E),(\tilde{\phi}, E) \in \mu$

2. the union of any number of soft sets in $\mu$ belongs to $\mu$.
Let $X$ be an initial universe set and $E$ be a set of parameters. The set of all soft points on the set $X$ is denoted by $S S(X)_{E}$. It is obvious that each soft set can be expressed as a union of soft points. For this reason, to give the family of all soft sets on $X$ it is sufficient to give only soft points on $X$.

Definition 2.22 [8] A quadrable system $\left(X, \tau_{1}, \tau_{2}, E\right)$ is called a soft bitopological space, where $\tau_{1}, \tau_{2}$ are arbitrary soft topologies on $X$ and $E$ be set of parameters.

Definition 2.23 [9] Let $\left(X, \tau_{1}, \tau_{2}, E\right)$ be a soft bitopological space. A soft set $(G, E)$ over $X$ is said to be a soft pair-wise open set in $\left(X, \tau_{1}, \tau_{2}, E\right)$ (soft popen set, for short) if there exists a soft open set $\left(G_{1}, E\right)$ in $\tau_{1}$ and a soft open set $\left(G_{2}, E\right)$ in $\tau_{2}$ such that $(G, E)=\left(G_{1}, E\right) \widetilde{\cup}\left(G_{2}, E\right)$.

Definition 2.24 [9] Let $\left(X, \tau_{1}, \tau_{2}, E\right)$ be a soft bitopological space. A soft set $(G, E)$ over $X$ is said to be a soft pair-wise closed set in $\left(X, \tau_{1}, \tau_{2}, E\right)$ (soft pclosed set, for short) if its complement is a soft p-

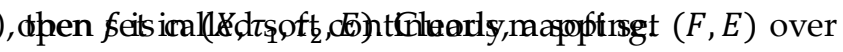
$X$ is a soft p-closed set in $\left(X, \tau_{1}, \tau_{2}, E\right)$ if there exists a soft closed set $\left(F_{1}, E\right)$ in $\tau_{1}^{c}$ and a soft closed set $\left(F_{2}, E\right)$ in $\tau_{2}^{c}$ such that $(F, E)=\left(F_{1}, E\right) \widetilde{\cap}\left(F_{2}, E\right)$, where

$$
\tau_{i}^{c}=\left\{(G, E)^{c} \in S S(X)_{E}:(G, E) \in \tau_{i}\right\}, i=1,2 .
$$

The family of all soft p-open (closed) sets in $\left(X, \tau_{1}, \tau_{2}, E\right)$ is denoted by $\operatorname{POS}\left(X, \tau_{1}, \tau_{2}\right)_{E}$ $\left(P C S\left(X, \tau_{1}, \tau_{2}\right)_{E}\right)$, respectively.

Theorem 2.1 [9] Let $\left(X, \tau_{1}, \tau_{2}, E\right)$ be a soft bitopological space. Then,

1. $(\tilde{X}, E),(\tilde{\phi}, E)$ are soft p-open sets and soft p-closed sets.

2. An arbitrary union of soft p-open sets is a soft popen set.

3. An arbitrary intersection soft p-closed sets is a soft p-closed set.

4. If $(G, E) \widetilde{\in} \tau_{1} \widetilde{\cap} \tau_{2}$ and $(H, E) \widetilde{\in} \operatorname{POS}\left(X, \tau_{1}, \tau_{2}\right)_{E}$, then $(G, E) \widetilde{\cap}(H, E) \widetilde{\in} P O S\left(X, \tau_{1}, \tau_{2}\right)_{E}$.

Corollary 2.1 [9] Let $\left(X, \tau_{1}, \tau_{2}, E\right)$ be a soft bitopological space. Then, the family of all soft $\mathrm{p}$ - 
open soft sets is a soft supratopology on $X$. This soft supratopology we denoted by $\tau_{12}$, i.e.,

$$
\begin{aligned}
\tau_{12} & =P O S\left(X, \tau_{1}, \tau_{2}\right)_{E} \\
& =\left\{(G, E)=\left(G_{1}, E\right) \widetilde{U}\left(G_{2}, E\right):\left(G_{i}, E\right) \in \tau_{i}, i=1,2\right\},
\end{aligned}
$$

and the triple $\left(X, \tau_{12}, E\right)$ is the soft supratopological spaces associated to the soft bitopological space $\left(X, \tau_{1}, \tau_{2}, E\right)$.

Theorem 2.2 [9] Let $\left(X, \tau_{1}, \tau_{2}, E\right)$ be a soft bitopological space. Then,

1. every soft $\tau_{i}$-open set is a soft p-open soft set, $i=1,2$

2. every soft $\tau_{i}$-closed set is a soft p-closed soft set, $i=1,2$

Definition 2.25 [9] Let $\left(X, \tau_{1}, \tau_{2}, E\right)$ be a soft bitopological space and $(G, E) \widetilde{\in} S S(X)_{E}$. The soft pair-wise closure of $(G, E)$, denoted by $c l_{p}^{S}(G, E)$, is the intersection of all soft p-closed super sets of $(G, E)$, i.e.,

$$
c l_{p}^{S}(G, E)=\widetilde{\cap}\left\{(F, E) \in \tau_{12}^{c}:(G, E) \widetilde{\subseteq}(F, E)\right\} .
$$

Clearly, $\operatorname{cl}_{p}^{S}(G, E)$ is the smallest soft p-closed set containing $(G, E)$.

Definition 2.26 [9] Let $\left(X, \tau_{1}, \tau_{2}, E\right)$ be a soft bitopological space and $(G, E) \widetilde{\in} S S(X)_{E}$. The soft pair-wise interior of $(G, E)$, denoted by $\operatorname{int}_{p}^{S}(G, E)$, is the union of all soft p-open subsets of $(G, E)$, i.e.,

$$
\operatorname{int}_{p}^{S}(G, E)=\widetilde{U}\left\{(F, E) \in \tau_{12}:(F, E) \widetilde{\subseteq}(G, E)\right\} .
$$

Clearly, $\operatorname{int}_{p}^{S}(G, E)$ is the largest soft p-open set contained in $(G, E)$.

\section{Soft Pair-Wise Neighborhood System}

Definition 3.1 Let $\left(X, \tau_{1}, \tau_{2}, E\right)$ be a soft bitopological space, $(F, E)$ be a soft set over $X$ and $x_{e} \widetilde{\in} S S(X)_{E}$. Then $(F, E)$ is said to be a soft pair-wise neighborhood (briefly soft p-nbd) of $x_{e}$, if there exists a soft p-open set $(G, E)$ such that $x_{e} \widetilde{\in}(G, E) \widetilde{\subseteq}(F, E)$.

Definition 3.2 Let $\left(X, \tau_{1}, \tau_{2}, E\right)$ be a soft bitopological space and $(H, E)$ be a soft set over $X$. A soft set $(F, E)$ over $(X, E)$ is said to be a soft p-nbd of the soft set
$(H, E)$ if $(F, E)$ is soft p-nbd of each soft point of $(H, E)$.

Theorem 3.1 A soft set $(F, E)$ over $X$ is soft p-open iff $(F, E)$ is a soft p-nbd of each its soft points.

Proof. Let $(F, E)$ be a soft p-open set and $x_{e} \widetilde{\in}(F, E)$. Then $x_{e} \widetilde{\in}(F, E) \widetilde{\subseteq}(F, E)$. Therefore $(F, E)$ is a soft pnbd of $x_{e}$ for each $x_{e} \widetilde{\in}(F, E)$.

Conversly, suppose that $(F, E)$ is a soft p-nbd of its each soft points and $x_{e} \widetilde{\in}(F, E)$. Then there exists soft p-open set $(G, E)$ such that $x_{e} \widetilde{\in}(G, E) \widetilde{\subseteq}(F, E)$. Since

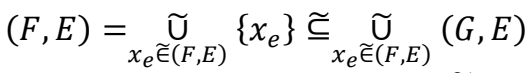

$$
\begin{aligned}
& \widetilde{\widetilde{\Xi}} \underset{x_{e} \widetilde{\in}(F, E)}{\widetilde{G}}(F, E)=(F, E)
\end{aligned}
$$

it follow that $(F, E)$ is a union of soft p-open sets. Hence $(F, E)$ is p-open soft set.

Let $\left(X, \tau_{1}, \tau_{2}, E\right)$ be a soft bitopological space and $x_{e} \widetilde{\in} S S(X)_{E}$. The family of all soft p-nbds of soft point $x_{e}$ denoted by $N_{\tau_{12}}\left(x_{e}\right)$.

Proposition 3.1 If $\left\{N_{\tau_{12}}\left(x_{e}\right): x_{e} \widetilde{\in} S S(X)_{E}\right\}$ is a system of soft p-nbds, then

1. For every $(F, E) \in N_{\tau_{12}}\left(x_{e}\right), x_{e} \widetilde{\in}(F, E)$;

2. $(F, E) \in N_{\tau_{12}}\left(x_{e}\right)$ and $(F, E) \widetilde{\subseteq}(G, E) \Rightarrow(G, E) \in$ $N_{\tau_{12}}\left(x_{e}\right)$;

3. $(F, E) \in N_{\tau_{12}}\left(x_{e}\right) \Rightarrow \exists(G, E) \in N_{\tau_{12}}\left(x_{e}\right)$ such that $(G, E) \widetilde{\subseteq}(F, E)$ and $(G, E) \in N_{\tau_{12}}\left(y_{e}{ }^{\prime}\right)$, for every $y_{e}, \widetilde{\in}(G, E)$.

Proof. Proofs of 1 and 2 are straightforward.

3. Let $(F, E)$ be a soft p-nbd of $x_{e}$, then there exists a soft p-open set $(G, E) \in \tau_{12} \quad$ such that $x_{e} \widetilde{\in}(G, E) \widetilde{\subseteq}(F, E)$. Since $x_{e} \widetilde{\in}(G, E) \widetilde{\subseteq}(G, E)$ can be written, then $(G, E) \in N_{\tau_{12}}\left(x_{e}\right)$. From the Theorem 3.1, if $(G, E)$ is soft p-open set then $(G, E)$ is a soft pnbd of its each soft points, i.e., $(G, E) \in N_{\tau_{12}}\left(y_{e}{ }^{\prime}\right)$, for every $y_{e}, \widetilde{\in}(G, E)$.

Remark 3.1 Generally, $(F, E),(G, E) \in N_{\tau_{12}}\left(x_{e}\right) \Rightarrow$ $(F, E) \widetilde{\cap}(G, E) \notin N_{\tau_{12}}\left(x_{e}\right)$. Actually, if $(F, E)$ and $(G, E) \in N_{\tau_{12}}\left(x_{e}\right)$, there exist $\left(U_{1}, E\right),\left(U_{2}, E\right) \in \tau_{12}$ such that $x_{e} \widetilde{\in}\left(U_{1}, E\right) \widetilde{\subseteq}(F, E)$ and $x_{e} \widetilde{\in}\left(U_{2}, E\right) \widetilde{\subseteq}(G, E)$. 
But $\left(U_{1}, E\right) \widetilde{\cap}\left(U_{2}, E\right)$ need not be a soft p-open set. Therefore, $(F, E) \widetilde{\cap}(G, E)$ need not be a soft p-nbd.

Proposition 3.2 Let $\left(X, \tau_{1}, \tau_{2}, E\right)$ be a soft bitopological space. Then

$$
N_{\tau_{12}}\left(x_{e}\right)=N_{\tau_{1}}\left(x_{e}\right) \widetilde{\cup} N_{\tau_{2}}\left(x_{e}\right)
$$

for each $x_{e} \widetilde{\in} S S(X)_{E}$.

Proof. Let $x_{e} \widetilde{\in} S S(X)_{E}$ be any soft point and $(F, E) \in$ $N_{\tau_{12}}\left(x_{e}\right)$ be any soft p-nbd of $x_{e}$. Then there exists a soft p-open $(G, E) \in \tau_{12}$ such that $x_{e} \widetilde{\in}(G, E) \widetilde{\subseteq}(F, E)$. If $(G, E) \in \tau_{12}$, there exist $\left(G_{1}, E\right) \in \tau_{1}$ and $\left(G_{2}, E\right) \in \tau_{2}$ such that $(G, E)=\left(G_{1}, E\right) \widetilde{\cup}\left(G_{2}, E\right)$. Since $x_{e} \widetilde{\in}(G, E)=\left(G_{1}, E\right) \widetilde{U}\left(G_{2}, E\right)$, then $x_{e} \widetilde{\in}\left(G_{1}, E\right)$ or $x_{e} \widetilde{\in}\left(G_{2}, E\right)$. So $x_{e} \widetilde{\in}\left(G_{1}, E\right) \widetilde{\subseteq}(G, E) \widetilde{\subseteq}(F, E)$ or $x_{e} \widetilde{\in}\left(G_{2}, E\right) \widetilde{\subseteq}(G, E) \widetilde{\subseteq}(F, E)$. In this case, $(F, E) \in$ $N_{\tau_{1}}\left(x_{e}\right) \quad$ or $\quad(F, E) \in N_{\tau_{2}}\left(x_{e}\right), \quad$ i. $\quad$ e., $\quad(F, E) \in$ $N_{\tau_{1}}\left(x_{e}\right) \widetilde{\cup} N_{\tau_{2}}\left(x_{e}\right)$.

Conversly, suppose that $(F, E) \in N_{\tau_{1}}\left(x_{e}\right) \widetilde{\cup} N_{\tau_{2}}\left(x_{e}\right)$. Then $(F, E) \in N_{\tau_{1}}\left(x_{e}\right)$ or $(F, E) \in N_{\tau_{2}}\left(x_{e}\right)$. Hence, there exists $x_{e} \widetilde{\in}\left(G_{1}, E\right) \in \tau_{1}$ or $x_{e} \widetilde{\in}\left(G_{2}, E\right) \in \tau_{2}$ such that $x_{e} \widetilde{\in}\left(G_{1}, E\right) \widetilde{\subseteq}(F, E)$ and $x_{e} \widetilde{\in}\left(G_{2}, E\right) \widetilde{\subseteq}(F, E)$. As a result, $x_{e} \widetilde{\in}\left(G_{1}, E\right) \widetilde{\cup}\left(G_{2}, E\right)=(G, E) \widetilde{\subseteq}(F, E)$ such that $(G, E) \in \tau_{12}$ i. e., $(F, E) \in N_{\tau_{12}}\left(x_{e}\right)$.

Example 3.1 Let $X=\left\{x^{1}, x^{2}, x^{3}\right\}$ and $E=\left\{e_{1}, e_{2}\right\}$. We consider the following soft sets over $X$;

$\left(F_{1}, E\right)=\left\{\left(e_{1},\left\{x^{1}, x^{2}\right\}\right),\left(e_{2},\left\{x^{2}, x^{3}\right\}\right)\right\}$,

$\left(F_{2}, E\right)=\left\{\left(e_{1},\left\{x^{2}\right\}\right),\left(e_{2},\left\{x^{1}, x^{2}\right\}\right)\right\}$,

$\left(F_{3}, E\right)=\left\{\left(e_{1},\left\{x^{2}\right\}\right),\left(e_{2},\left\{x^{2}\right\}\right)\right\}$,

$\left(F_{4}, E\right)=\left\{\left(e_{1},\left\{x^{1}, x^{2}\right\}\right),\left(e_{2}, X\right)\right\}$,

$\left(G_{1}, E\right)=\left\{\left(e_{1},\left\{x^{1}\right\}\right),\left(e_{2},\left\{x^{1}, x^{2}\right\}\right)\right\}$,

$\left(G_{2}, E\right)=\left\{\left(e_{1},\left\{x^{2}, x^{3}\right\}\right),\left(e_{2},\left\{x^{2}, x^{3}\right\}\right)\right\}$,

$\left(G_{3}, E\right)=\left\{\left(e_{1}, \emptyset\right),\left(e_{2},\left\{x^{2}\right\}\right)\right\}$.

Then, $\left(X, \tau_{1}, \tau_{2}, E\right)$ is a soft bitopological space, where

$\tau_{1}=\left\{\Phi, \tilde{X},\left(F_{1}, E\right),\left(F_{2}, E\right),\left(F_{3}, E\right),\left(F_{4}, E\right)\right\}$ and

$\tau_{2}=\left\{\Phi, \tilde{X},\left(G_{1}, E\right),\left(G_{2}, E\right),\left(G_{3}, E\right)\right\}$.

It is clear that,

$$
\tau_{12}=\left\{\begin{array}{l}
\Phi, \tilde{X},\left(F_{1}, E\right),\left(F_{2}, E\right),\left(F_{3}, E\right),\left(F_{4}, E\right), \\
\left(G_{1}, E\right),\left(G_{2}, E\right),\left(G_{3}, E\right),\left(H_{1}, E\right),\left(H_{2}, E\right),\left(H_{3}, E\right)
\end{array}\right\},
$$

Where,

$$
\begin{aligned}
\left(H_{1}, E\right) & =\left(F_{1}, E\right) \widetilde{U}\left(G_{2}, E\right)=\left\{\left(e_{1}, X\right),\left(e_{2},\left\{x^{2}, x^{3}\right\}\right)\right\} \\
\left(H_{2}, E\right) & =\left(F_{2}, E\right) \widetilde{\cup}\left(G_{1}, E\right) \\
& =\left\{\left(e_{1},\left\{x^{1}, x^{2}\right\}\right),\left(e_{2},\left\{x^{1}, x^{2}\right\}\right)\right\} \\
\left(H_{3}, E\right) & =\left(F_{2}, E\right) \widetilde{\cup}\left(G_{2}, E\right)=\left\{\left(e_{1},\left\{x^{2}, x^{3}\right\}\right),\left(e_{2}, X\right)\right\} .
\end{aligned}
$$

Consequently, for arbitrary soft point $x_{e_{1}}^{1} \widetilde{\in} S S(X)_{E}$

$$
\begin{aligned}
& N_{\tau_{12}}\left(x_{e_{1}}^{1}\right) \\
& =\left\{\begin{array}{l}
\tilde{X},\left(F_{1}, E\right),\left(F_{4}, E\right),\left(G_{1}, E\right),\left(H_{1}, E\right),\left(H_{2}, E\right), \\
\left\{\left(e_{1},\left\{x^{1}\right\}\right),\left(e_{2}, X\right)\right\},\left\{\left(e_{1}, X\right),\left(e_{2},\left\{x^{1}, x^{2}\right\}\right)\right\}
\end{array}\right\} .
\end{aligned}
$$

On the other hand,

$N_{\tau_{1}}\left(x_{e_{1}}^{1}\right)=\left\{\tilde{X},\left(F_{1}, E\right),\left(F_{4}, E\right),\left(H_{1}, E\right)\right\}$,

$N_{\tau_{2}}\left(x_{e_{1}}^{1}\right)=\left\{\begin{array}{l}\tilde{X},\left(F_{4}, E\right),\left(G_{1}, E\right),\left(H_{2}, E\right), \\ \left\{\left(e_{1},\left\{x^{1}\right\}\right),\left(e_{2}, X\right)\right\},\left\{\left(e_{1}, X\right),\left(e_{2},\left\{x^{1}, x^{2}\right\}\right)\right\}\end{array}\right\}$.

Therefore, $N_{\tau_{1}}\left(x_{e_{1}}^{1}\right) \widetilde{\cup} N_{\tau_{2}}\left(x_{e_{1}}^{1}\right)=N_{\tau_{12}}\left(x_{e_{1}}^{1}\right)$.

Definition 3.3 An operator $\varphi^{S}: S S(X)_{E} \rightarrow S S(X)_{E}$ is called a soft supra neighborhood operator if it satisfies the following conditions for all soft sets $(G, E),(H, E) \in S S(X)_{E}$

$\varphi_{1}^{S}: \forall(F, E) \in \varphi^{S}\left(x_{e}\right), x_{e} \widetilde{\epsilon}(F, E)$

$\varphi_{2}^{S}:(F, E) \in \varphi^{S}\left(x_{e}\right)$ and $(F, E) \widetilde{\subseteq}(G, E) \Rightarrow(G, E) \in$ $\varphi^{S}\left(x_{e}\right)$;

$\varphi_{3}^{S}:(F, E) \in \varphi^{S}\left(x_{e}\right) \Rightarrow \exists(G, E) \in \varphi^{S}\left(x_{e}\right)$ such that $(F, E) \widetilde{\subseteq}(G, E)$ and $(G, E) \in \varphi^{s}\left(y_{e}{ }^{\prime}\right), y_{e}, \widetilde{\in}(G, E)$.

Theorem 3.2 Let $\left(X, \tau_{1}, \tau_{2}, E\right)$ be a soft bitopological space. Then the operator $\varphi^{S}: S S(X)_{E} \rightarrow S S(X)_{E}$ which defined by

$$
\varphi_{\tau_{12}}^{s}\left(x_{e}\right)=\varphi_{\tau_{1}}^{s}\left(x_{e}\right) \widetilde{\cup} \varphi_{\tau_{2}}^{s}\left(x_{e}\right)
$$

is a soft supra neighborhood operator and it is induced a unique soft supratopology given by $\left\{(F, E) \in S S(X)_{E}: \forall x_{e} \widetilde{\in}(F, E)\right.$ for $\left.(F, E) \in \varphi_{\tau_{12}}^{S}\left(x_{e}\right)\right\}$ which exactly $\tau_{12}$.

Proof. Straightforward.

Definition 3.4 Let $\left(X, \tau_{1}, \tau_{2}, E\right)$ be a soft bitopological space and $(G, E) \in S S(X)_{E}$. Then $x_{e} \widetilde{\in} S S(X)_{E}$ is called soft pair-wise limit point of $(G, E)$ if $((G, E) \backslash$ $\left.x_{e}\right) \widetilde{\cap}(F, E) \neq \emptyset$ for every soft p-open set $(F, E)$. The set of all soft pair-wise limit points of $(G, E)$ is called 
soft p-derived set and denoted by $d_{p}^{s}((G, E))$.

Theorem 3.3 Let $\left(X, \tau_{1}, \tau_{2}, E\right)$ be a soft bitopological space and $(F, E),(G, E) \in S S(X)_{E}$. Then

1. $c l_{p}^{s}(F, E)=(F, E) \widetilde{\cup} d_{p}^{s}((F, E))$;

2. $(F, E) \widetilde{\subseteq}(G, E) \Rightarrow d_{p}^{s}((F, E)) \widetilde{\subseteq} d_{p}^{s}((G, E))$;

3. $d_{p}^{s}((F, E)) \widetilde{\cup} d_{p}^{s}((G, E)) \widetilde{\subseteq} d_{p}^{s}((F, E) \widetilde{\cup}(G, E))$.

Proof. Straightforward.

Remark 3.2 The equality of the Theorem 5.(3) is not true in general as shown in the following examples.

Example 3.2 Let $X=\left\{x^{1}, x^{2}, x^{3}\right\}$ and $E=\{e\}$. We consider the following soft sets over $X$;

$\left(F_{1}, E\right)=\left\{\left(e,\left\{x^{1}, x^{2}\right\}\right)\right\}$,

$\left(F_{2}, E\right)=\left\{\left(e,\left\{x^{3}\right\}\right)\right\}$,

$\left(G_{1}, E\right)=\left\{\left(e,\left\{x^{1}, x^{3}\right\}\right)\right\}$,

$\left(G_{2}, E\right)=\left\{\left(e,\left\{x^{2}, x^{3}\right\}\right)\right\}$,

$\left(G_{3}, E\right)=\left\{\left(e,\left\{x^{3}\right\}\right)\right\}$.

Then $\left(X, \tau_{1}, \tau_{2}, E\right)$ is a soft bitopological space, where

$\tau_{1}=\left\{\Phi, \tilde{X},\left(F_{1}, E\right),\left(F_{2}, E\right)\right\}$ and

$\tau_{2}=\left\{\Phi, \tilde{X},\left(G_{1}, E\right),\left(G_{2}, E\right),\left(G_{3}, E\right)\right\}$.

It is clear that,

$$
\tau_{12}=\left\{\Phi, \tilde{X},\left(F_{1}, E\right),\left(F_{2}, E\right),\left(G_{1}, E\right),\left(G_{2}, E\right),\left(G_{3}, E\right)\right\} .
$$

Now, let $(H, E)=\left\{\left(e,\left\{x^{2}, x^{3}\right\}\right)\right\} \quad$ and $\quad(K, E)=$ $\left\{\left(e,\left\{x^{1}\right\}\right)\right\}$ be two soft set over $X$. Then

$d_{p}^{s}((H, E))=\left\{\left(e,\left\{x^{1}\right\}\right)\right\}$,

$d_{p}^{s}((K, E))=\Phi$,

$d_{p}^{s}((H, E) \widetilde{\cup}(K, E))=\left\{\left(e,\left\{x^{1}, x^{2}\right\}\right)\right\}$.

Therefore,

$$
d_{p}^{s}((F, E)) \widetilde{\cup} d_{p}^{s}((G, E)) \neq d_{p}^{s}((F, E) \widetilde{\cup}(G, E))
$$

\section{Soft Pair-Wise Continuity}

We introduce a different kind of a definition of soft continuity in a soft bitopological space, based on the definition of a soft point given in [3].

Definition 4.1 Let $\left(X, \tau_{1}, \tau_{2}, E\right)$ and $\left(Y, \eta_{1}, \eta_{2}, E\right)$ be two soft bitopological spaces and $\left(f, 1_{E}\right):\left(X, \tau_{1}, \tau_{2}, E\right) \rightarrow\left(Y, \eta_{1}, \eta_{2}, E\right)$ (briefly denoted by $f)$ be a soft mapping. For each soft p-nbd $(G, E)$ of $f\left(x_{e}\right)$, if there exists a soft p-nbd $(F, E)$ of soft point $x_{e} \widetilde{\in} S S(X)_{E}$ such that $f((F, E)) \widetilde{\subseteq}(G, E)$, then $f$ is said to be soft pair-wise continuous mapping (soft pcontinuous mapping, briefly) at $x_{e}$.

If $f$ is soft p-continuous mapping for all $x_{e} \widetilde{\in} S S(X)_{E}$, then $f$ is called soft p-continuous mapping.

Example 4.1 Let $X=\left\{x^{1}, x^{2}, x^{3}\right\}, Y=\left\{y^{1}, y^{2}, y^{3}\right\}$ and $E=\left\{e_{1}, e_{2}\right\}$. Then $\tau_{1}=\left\{\Phi, \tilde{X},\left(F_{1}, E\right),\left(F_{2}, E\right),\left(F_{3}, E\right)\right\}$, $\tau_{2}=\left\{\Phi, \tilde{X},\left(G_{1}, E\right),\left(G_{2}, E\right),\left(G_{3}, E\right),\left(G_{4}, E\right)\right\}$ are two soft topological spaces over $X$ and $\eta_{1}=$ $\left\{\Phi, \tilde{Y},\left(H_{1}, E\right),\left(H_{2}, E\right),\left(H_{3}, E\right)\right\}, \quad \eta_{2}=$ $\left\{\Phi, \tilde{Y},\left(K_{1}, E\right),\left(K_{2}, E\right),\left(K_{3}, E\right)\right\}$ are two soft topological spaces over $Y$. Here the soft sets over $X$ and $Y$ defined as follows:

$\left(F_{1}, E\right)=\left\{\left(e_{1},\left\{x^{1}, x^{2}\right\}\right),\left(e_{2},\left\{x^{2}, x^{3}\right\}\right)\right\} ;$

$\left(F_{2}, E\right)=\left\{\left(e_{1},\left\{x^{1}, x^{3}\right\}\right),\left(e_{2},\left\{x^{1}\right\}\right)\right\}$

$\left(F_{3}, E\right)=\left\{\left(e_{1},\left\{x^{1}\right\}\right),\left(e_{2}, \varnothing\right)\right\}$;

$\left(G_{1}, E\right)=\left\{\left(e_{1},\left\{x^{2}\right\}\right),\left(e_{2},\left\{x^{1}, x^{2}\right\}\right)\right\}$

$\left(G_{2}, E\right)=\left\{\left(e_{1},\left\{x^{1}, x^{3}\right\}\right),\left(e_{2},\left\{x^{1}, x^{2}\right\}\right)\right\}$;

$\left(G_{3}, E\right)=\left\{\left(e_{1}, \emptyset\right),\left(e_{2},\left\{x^{1}, x^{2}\right\}\right)\right\} ;$

$\left(G_{4}, E\right)=\left\{\left(e_{1}, X\right),\left(e_{2},\left\{x^{1}, x^{2}\right\}\right)\right\}$;

and

$\left(H_{1}, E\right)=\left\{\left(e_{1},\left\{y^{1}, y^{3}\right\}\right),\left(e_{2},\left\{y^{1}, y^{2}\right\}\right)\right\}$

$\left(H_{2}, E\right)=\left\{\left(e_{1}, Y\right),\left(e_{2},\left\{y^{3}\right\}\right)\right\}$;

$\left(H_{3}, E\right)=\left\{\left(e_{1},\left\{y^{1}, y^{3}\right\}\right),\left(e_{2}, \varnothing\right)\right\}$;

$\left(K_{1}, E\right)=\left\{\left(e_{1},\left\{y^{1}\right\}\right),\left(e_{2},\left\{y^{1}, y^{3}\right\}\right)\right\}$;

$\left(K_{2}, E\right)=\left\{\left(e_{1},\left\{y^{2}, y^{3}\right\}\right),\left(e_{2},\left\{y^{1}, y^{2}\right\}\right)\right\}$;

$\left(K_{3}, E\right)=\left\{\left(e_{1}, \emptyset\right),\left(e_{2},\left\{y^{1}\right\}\right)\right\}$.

Then $\left(X, \tau_{1}, \tau_{2}, E\right)$ and $\left(Y, \eta_{1}, \eta_{2}, E\right)$ are two soft bitopological spaces where

$$
\tau_{12}=\left\{\begin{array}{l}
\Phi, \tilde{X},\left(F_{1}, E\right),\left(F_{2}, E\right),\left(F_{3}, E\right),\left(G_{1}, E\right),\left(G_{2}, E\right),\left(G_{3}, E\right),\left(G_{4}, E\right), \\
\left(U_{1}, E\right)=\left(F_{1}, E\right) \widetilde{U}\left(G_{1}, E\right)=\left\{\left(e_{1},\left\{x^{1}, x^{2}\right\}\right),\left(e_{2}, X\right)\right\}, \\
\left(U_{2}, E\right)=\left(F_{3}, E\right) \widetilde{U}\left(G_{1}, E\right)=\left\{\left(e_{1},\left\{x^{1}, x^{2}\right\}\right),\left(e_{2},\left\{x^{1}, x^{2}\right\}\right),\right\}, \\
\left(U_{3}, E\right)=\left(F_{3}, E\right) \widetilde{U}\left(G_{3}, E\right)=\left\{\left(e_{1},\left\{x^{1}\right\}\right),\left(e_{2},\left\{x^{1}, x^{2}\right\}\right),\right\}
\end{array}\right\}
$$

and

$$
\eta_{12}=\left\{\begin{array}{l}
\Phi, \tilde{Y},\left(H_{1}, E\right),\left(H_{2}, E\right),\left(H_{3}, E\right),\left(K_{1}, E\right),\left(K_{2}, E\right),\left(K_{3}, E\right), \\
\left(V_{1}, E\right)=\left(H_{1}, E\right) \widetilde{U}\left(K_{1}, E\right)=\left\{\left(e_{1},\left\{y^{1}, y^{3}\right\}\right),\left(e_{2}, Y\right)\right\}, \\
\left(V_{2}, E\right)=\left(H_{1}, E\right) \widetilde{U}\left(K_{2}, E\right)=\left\{\left(e_{1}, Y\right),\left(e_{2},\left\{y^{1}, y^{2}\right\}\right)\right\}, \\
\left(V_{3}, E\right)=\left(H_{2}, E\right) \widetilde{U}\left(K_{3}, E\right)=\left\{\left(e_{1}, Y\right),\left(e_{2},\left\{y^{1}, y^{3}\right\}\right)\right\}, \\
\left(V_{4}, E\right)=\left(H_{3}, E\right) \widetilde{U}\left(K_{1}, E\right)=\left\{\left(e_{1},\left\{y^{1}, y^{3}\right\}\right),\left(e_{2},\left\{y^{1}, y^{3}\right\}\right)\right\}, \\
\left(V_{5}, E\right)=\left(H_{3}, E\right) \widetilde{U}\left(K_{3}, E\right)=\left\{\left(e_{1},\left\{y^{1}, y^{3}\right\}\right),\left(e_{2},\left\{y^{1}\right\}\right)\right\}
\end{array}\right\} .
$$


If the mapping $f: X \rightarrow Y$ defined as $f\left(x_{e_{j}}^{i}\right)=y_{e_{j}}^{i} i=$ $\overline{1,3}, j=1,2$, then $f$ is soft $\mathrm{p}$-continuous mapping at $x_{e_{1}}^{1}$ and is not soft p-continuous mapping at $x_{e_{1}}^{3}$. Therefore $f$ is not soft p-continuous mapping on $X$.

Theorem 4.1 Let $\left(X, \tau_{1}, \tau_{2}, E\right)$ and $\left(Y, \eta_{1}, \eta_{2}, E\right)$ be two soft bitopological spaces and $f:\left(X, \tau_{1}, \tau_{2}, E\right) \rightarrow$ $\left(Y, \eta_{1}, \eta_{2}, E\right)$ be a soft mapping. Then $f$ is a soft $\mathrm{p}$ continuous mapping iff $f^{-1}((G, E)) \in \tau_{12}$ is soft popen set, for each soft p-open set $(G, E) \in \eta_{12}$.

Proof. Suppose that $f$ is a soft $\mathrm{p}$-continuous mapping and $(G, E) \in \eta_{12}$. Let us show that $f^{-1}((G, E)) \in \tau_{12}$. For every soft point $x_{e} \widetilde{\in} f^{-1}((G, E))$ since $f\left(x_{e}\right) \widetilde{\in}(G, E)$ and $f$ is a soft p-continuous mapping, then there exists a soft p-nbd $(F, E)$ of the soft point $x_{e}$ such that $f((F, E)) \widetilde{\subseteq}(G, E)$. Therefore, $x_{e} \widetilde{\epsilon}(F, E) \widetilde{\subseteq} f^{-1}((G, E))$ and $f^{-1}((G, E))$ is a soft popen set from Theorem 3.1 .

Conversely, let $x_{e}$ be a soft point over $X$ and $f\left(x_{e}\right) \widetilde{\epsilon}(G, E)$ be a soft p-open set in $Y$. Then $x_{e} \widetilde{\in} f^{-1}((G, E))$ is a soft p-open set and $f\left(f^{-1}((G, E))\right) \widetilde{\subseteq}(G, E)$. That is, $f$ is a soft $\mathrm{p}$ continuous mapping.

Theorem 4.2 Let $\left(X, \tau_{1}, \tau_{2}, E\right)$ and $\left(Y, \eta_{1}, \eta_{2}, E\right)$ be two soft bitopological spaces and $f:\left(X, \tau_{1}, \tau_{2}, E\right) \rightarrow$ $\left(Y, \eta_{1}, \eta_{2}, E\right)$ be a soft mapping. Then $f$ is soft $\mathrm{p}$ continuous iff $f^{-1}((G, E))$ soft p-closed in $X$ for every $(G, E) \widetilde{\in} S S(Y)_{E}$.

Proof. Let $f:\left(X, \tau_{1}, \tau_{2}, E\right) \rightarrow\left(Y, \eta_{1}, \eta_{2}, E\right)$ be a soft mapping and $(G, E)$ is any soft p-closed in $Y$. Since $(G, E)^{c}$ is soft p-open in $Y$ and $f$ is a soft pcontinuous mapping, then $f^{-1}\left((G, E)^{c}\right)$ is soft p-open set in $X$. Morover, $f^{-1}\left((G, E)^{c}\right)=\left(f^{-1}((G, E))\right)^{c}$. If $\left(f^{-1}((G, E))\right)^{c}$ is soft a soft p-open then $f^{-1}((G, E))$ is soft p-closed set in $X$.

Conversely, suppose that $f^{-1}((G, E))$ is soft p-closed set in $X$ whenever $(G, E)$ is soft p-closed in $Y$. For any soft p-open set $(H, E)$ over $Y,(H, E)^{c}$ is soft p-closed set. From the hypothesis, $f^{-1}\left((H, E)^{c}\right)$ is soft p-closed set in $X$. Since $f^{-1}\left((H, E)^{c}\right)=\left(f^{-1}((H, E))\right)^{c}$ and $\left(f^{-1}((H, E))\right)^{c}$ is soft p-closed set in $X$, then $f^{-1}((H, E))$ is soft open set in $X$. Therefore, $f$ is soft p-continuous.

Theorem 4.3 Let $\left(X, \tau_{1}, \tau_{2}, E\right)$ and $\left(Y, \eta_{1}, \eta_{2}, E\right)$ be two soft bitopological spaces and $f:\left(X, \tau_{1}, \tau_{2}, E\right) \rightarrow$ $\left(Y, \eta_{1}, \eta_{2}, E\right)$ be a soft mapping. Then $f$ is soft pcontinuous iff $f\left(c l_{p}^{s}((F, E))\right) \widetilde{\subseteq} c l_{p}^{s}(f((F, E)))$ for every $(F, E) \widetilde{\in} S S(X)_{E}$.

Proof. Let $f:\left(X, \tau_{1}, \tau_{2}, E\right) \rightarrow\left(Y, \eta_{1}, \eta_{2}, E\right)$ be a soft pcontinuous mapping and $(F, E) \widetilde{\in} S S(X)_{E}$. Since $c l_{p}^{s}(f((F, E)))$ is soft p-closed set in $Y$, $f^{-1}\left(c l_{p}^{s}(f((F, E)))\right)$ is soft p-closed in $X$ from the Theorem 4.2. Then

$c l_{p}^{s}\left(f^{-1}\left(c l_{p}^{s}(f((F, E)))\right)\right)=f^{-1}\left(c l_{p}^{s}(f((F, E)))\right)$.

We know that $f((F, E)) \widetilde{\subseteq} c l_{p}^{s}(f((F, E)))$, then $f((F, E)) \widetilde{\subseteq} f^{-1}(f((F, E))) \widetilde{\subseteq} f^{-1}\left(c l_{p}^{S}(f((F, E)))\right)$.

From 4.1,

$$
\begin{aligned}
c l_{p}^{s}((F, E)) & \widetilde{\subseteq} c l_{p}^{s}\left(f^{-1}\left(c l_{p}^{s}(f((F, E)))\right)\right) \\
& =f^{-1}\left(c l_{p}^{s}(f((F, E)))\right) .
\end{aligned}
$$

Therefore,

$$
f\left(c l_{p}^{s}((F, E))\right) \widetilde{\subseteq} l_{p}^{s}(f((F, E))) .
$$

Conversely, suppose that $f\left(c l_{p}^{S}((F, E))\right) \widetilde{\subseteq} c_{p}^{s}(f((F, E))) \quad$ for every $(F, E) \widetilde{\in} S S(X)_{E}$. Let $(G, E)$ be any soft p-closed set in $Y$, so that $c l_{p}^{S}((G, E))=(G, E)$. From the hypothesis,

$$
\begin{aligned}
f\left(c l_{p}^{s}\left(f^{-1}((G, E))\right)\right) & \widetilde{\subseteq} c l_{p}^{s}\left(f\left(f^{-1}((G, E))\right)\right) \\
& \widetilde{\subseteq} c l_{p}^{s}((G, E))=(G, E)
\end{aligned}
$$

is satisfied. Thus, $c l_{p}^{s}\left(f^{-1}((G, E))\right) \widetilde{\subseteq} f^{-1}((G, E))$ and $f^{-1}((G, E)) \widetilde{\subseteq} c l_{p}^{s}\left(f^{-1}((G, E))\right)$. That is, $c l_{p}^{s}\left(f^{-1}((G, E))\right)=f^{-1}((G, E))$ and so $f^{-1}((G, E))$ is a soft p-closed set in $X$. From the Theorem 4.2., it is clear that $f$ is soft $\mathrm{p}$-continuous mapping.

Theorem 4.4 Let $\left(X, \tau_{1}, \tau_{2}, E\right)$ and $\left(Y, \eta_{1}, \eta_{2}, E\right)$ be two soft bitopological spaces and $f:\left(X, \tau_{1}, \tau_{2}, E\right) \rightarrow$ $\left(Y, \eta_{1}, \eta_{2}, E\right)$ be a soft mapping. Then $f$ is a soft pcontinuous mapping iff $f:\left(X, \tau_{1}, E\right) \rightarrow\left(Y, \eta_{1}, E\right)$ and 
$f:\left(X, \tau_{2}, E\right) \rightarrow\left(Y, \eta_{2}, E\right) \quad$ are soft continuous mappings.

Proof. Let $f$ be a soft p-continuous mapping and $(G, E)$ be soft p-open set over $Y$. Then there exist $\left(G_{1}, E\right) \in \eta_{1} \quad$ and $\quad\left(G_{2}, E\right) \in \eta_{2} \quad$ such that $\left(G_{1}, E\right) \widetilde{\cup}\left(G_{2}, E\right)=(G, E)$. Since $f$ is a soft pcontinuous mapping, then

$$
\begin{aligned}
f^{-1}((G, E))=f^{-1}\left(\left(G_{1}, E\right) \widetilde{\cup}\left(G_{2}, E\right)\right) \\
=f^{-1}\left(\left(G_{1}, E\right)\right) \widetilde{\cup} f^{-1}\left(\left(G_{2}, E\right)\right)
\end{aligned}
$$

is soft p-open set. In this case, $f^{-1}\left(\left(G_{1}, E\right)\right) \in \tau_{1}$ and $f^{-1}\left(\left(G_{2}, E\right)\right) \in \tau_{2}$. That is, $f:\left(X, \tau_{1}, E\right) \rightarrow\left(Y, \eta_{1}, E\right)$ and $f:\left(X, \tau_{2}, E\right) \rightarrow\left(Y, \eta_{2}, E\right) \quad$ are soft continuous mappings.

Conversely, suppose that $f:\left(X, \tau_{1}, E\right) \rightarrow\left(Y, \eta_{1}, E\right)$ and $f:\left(X, \tau_{2}, E\right) \rightarrow\left(Y, \eta_{2}, E\right)$ is soft continuous mappings and let $\left(G_{1}, E\right) \in \eta_{1},\left(G_{2}, E\right) \in \eta_{2}$ be two soft open sets. Then there exists a soft p-open set $(G, E)$ such that $(G, E)=\left(G_{1}, E\right) \widetilde{\cup}\left(G_{2}, E\right)$. Since $\quad f:\left(X, \tau_{1}, E\right) \rightarrow$ $\left(Y, \eta_{1}, E\right)$ and $f:\left(X, \tau_{2}, E\right) \rightarrow\left(Y, \eta_{2}, E\right)$ is soft continuous mappings, then $f^{-1}\left(\left(G_{1}, E\right)\right) \in \tau_{1}$ and $f^{-1}\left(\left(G_{2}, E\right)\right) \in \tau_{2}$. Therefore, $f^{-1}\left(\left(G_{1}, E\right)\right) \widetilde{\cup} f^{-1}\left(\left(G_{2}, E\right)\right)=f^{-1}\left(\left(G_{1}, E\right) \widetilde{\cup}\left(G_{2}, E\right)\right)=$ $f^{-1}((G, E))$. That is, $f$ is a soft p-continuous mapping.

Theorem 4.5 Let $\left(X, \tau_{1}, \tau_{2}, E\right), \quad\left(Y, \eta_{1}, \eta_{2}, E\right)$ and $\left(Z, \sigma_{1}, \sigma_{2}, E\right)$ be soft topological spaces. If $f:\left(X, \tau_{1}, \tau_{2}, E\right) \rightarrow\left(Y, \eta_{1}, \eta_{2}, E\right)$ and $g:\left(Y, \eta_{1}, \eta_{2}, E\right) \rightarrow$ $\left(Z, \sigma_{1}, \sigma_{2}, E\right)$ are soft p-continuous, then $g \circ$ $f:\left(X, \tau_{1}, \tau_{2}, E\right) \rightarrow\left(Z, \sigma_{1}, \sigma_{2}, E\right)$ is soft p-continuous.

Proof. Let $(W, E) \in \sigma_{12}$ be a soft p-open and let us show that $(g \circ f)^{-1}((W, E))$ is a soft p-open set in $X$. Since $(g \circ f)^{-1}((W, E))=f^{-1}\left(g^{-1}((W, E))\right)$ and the mapping $g$ is soft p-continuous, then $g^{-1}((W, E))$ is sopft p-open set in $Y$. On the other hand, since $f$ is soft p-continuous then $f^{-1}\left(g^{-1}((W, E))\right)$ is sopt popen in $X$. That is, $(g \circ f)^{-1}((W, E))$ is soft p-open set in $X$ and $g \circ f$ is soft p-open continuous.

Definition 4.2 Let $\left(X, \tau_{1}, \tau_{2}, E\right)$ and $\left(Y, \eta_{1}, \eta_{2}, E\right)$ be two soft bitopological spaces and $f:\left(X, \tau_{1}, \tau_{2}, E\right) \rightarrow$ $\left(Y, \eta_{1}, \eta_{2}, E\right)$ be a soft mapping. Then, a: $f$ is called soft pair-wise open mapping (soft $\mathrm{p}$ open mapping, briefly) if $f((F, E))$ is soft p-open set in $Y$, for every soft p-open set $(F, E)$ in $X$;

b: $f$ is called soft pair-wise closed mapping (soft pclosed mapping, briefly) if $f((F, E))$ is soft p-closed set in $Y$, for every soft p-closed set $(F, E)$ in $X$.

Example 4.2 Let $X=\left\{x^{1}, x^{2}, x^{3}\right\}, Y=\left\{y^{1}, y^{2}, y^{3}\right\}$ and $E=\left\{e_{1}, \quad e_{2}\right\} . \quad$ Then $\quad \tau_{1}=\{\Phi, \tilde{X},(F, E)\}, \quad \tau_{2}=$ $\{\Phi, \tilde{X},(G, E)\}$ are two soft topological spaces over $X$ and $\eta_{1}=\{\Phi, \tilde{Y},(H, E)\}, \eta_{2}=\{\Phi, \tilde{Y},(K, E)\}$ are two soft topological spaces over $Y$. Here the soft sets over $X$ and $Y$ defined as follows:

$$
\begin{aligned}
& (F, E)=\left\{\left(e_{1},\left\{x^{1}, x^{3}\right\}\right),\left(e_{2},\left\{x^{2}, x^{3}\right\}\right)\right\} \\
& (G, E)=\left\{\left(e_{1},\left\{x^{2}, x^{3}\right\}\right),\left(e_{2},\left\{x^{1}, x^{3}\right\}\right)\right\}
\end{aligned}
$$

and

$$
\begin{aligned}
& (H, E)=\left\{\left(e_{1},\left\{y^{1}, y^{2}\right\}\right),\left(e_{2},\left\{y^{2}, y^{3}\right\}\right)\right\} \\
& (K, E)=\left\{\left(e_{1},\left\{y^{2}, y^{3}\right\}\right),\left(e_{2},\left\{y^{1}, y^{2}\right\}\right)\right\} .
\end{aligned}
$$

Then $\left(X, \tau_{1}, \tau_{2}, E\right)$ and $\left(Y, \eta_{1}, \eta_{2}, E\right)$ are two soft bitopological spaces. It is clear that, $\tau_{12}=$ $\{\Phi, \tilde{X},(F, E),(G, E)\}$ and $\eta_{12}=\{\Phi, \tilde{Y},(H, E),(K, E)\}$.

If the mapping $f: X \rightarrow Y$ defined as

$$
\begin{aligned}
& f\left(x_{e_{1}}^{1}\right)=y_{e_{1}}^{1}, f\left(x_{e_{2}}^{1}\right)=y_{e_{2}}^{1} \\
& f\left(x_{e_{1}}^{2}\right)=y_{e_{1}}^{3}, f\left(x_{e_{2}}^{2}\right)=y_{e_{2}}^{3} \\
& f\left(x_{e_{1}}^{3}\right)=y_{e_{1}}^{2}, f\left(x_{e_{2}}^{3}\right)=y_{e_{2}}^{2}
\end{aligned}
$$

then $f$ is both soft $\mathrm{p}$-open and soft p-closed mapping.

Theorem 4.6 Let $\left(X, \tau_{1}, \tau_{2}, E\right)$ and $\left(Y, \eta_{1}, \eta_{2}, E\right)$ be two soft bitopological spaces and $f:\left(X, \tau_{1}, \tau_{2}, E\right) \rightarrow$ $\left(Y, \eta_{1}, \eta_{2}, E\right)$ be a soft mapping. Then,

a: $f$ is soft p-open mapping iff $f\left(\operatorname{int}_{p}^{s}((F, E))\right) \widetilde{\widetilde{C}} \operatorname{int}_{p}^{s}(f((F, E)))$ for every $(F, E) \widetilde{\in} S S(X)_{E}$;

b: $f$ is soft p-closed mapping iff $c l_{p}^{s}(f((F, E))) \widetilde{\subseteq} f\left(c l_{p}^{s}((F, E))\right)$ for every $(F, E) \widetilde{\in} S S(X)_{E}$.

Proof. a: Let $f$ be a soft p-open mapping and $(F, E) \widetilde{\in} S S(X)_{E}$. We know that $\operatorname{int}_{p}^{S}((F, E))$ is soft popen and $\operatorname{int}_{p}^{S}((F, E)) \widetilde{\subseteq}(F, E)$. From the hypothesis, since $f$ is a soft p-open mapping then $f\left(\operatorname{int}_{p}^{S}((F, E))\right)$ is a soft p-open set in $Y$ and 
$f\left(\operatorname{int}_{p}^{s}((F, E))\right) \widetilde{\subseteq} f((F, E))$. In this case, Proof. It is easily obtained.

$f\left(\operatorname{int}_{p}^{s}((F, E))\right) \widetilde{\subseteq} \operatorname{int}_{p}^{S}(f((F, E)))$ is obtained.

\section{Conclusion}

Conversely, suppose that $(G, E)$ is any soft p-open set. Then $\operatorname{int}_{p}^{s}((G, E))=(G, E)$. From the hypothesis,

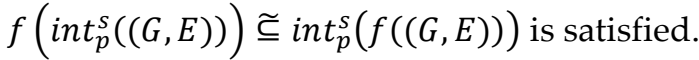

Therefore,

$$
\begin{aligned}
& f((G, E))=f\left(\operatorname{int}_{p}^{S}((G, E))\right) \\
& \widetilde{\subseteq} \operatorname{int}_{p}^{s}(f((G, E))) \widetilde{\subseteq} f((G, E)) \\
& \Rightarrow f((G, E))=\operatorname{int}_{p}^{s}(f((G, E))) \text {. }
\end{aligned}
$$

That is, $f((G, E))$ is a soft p-open set and so $f$ is soft p-open mapping.

$\mathrm{b}$ : The proof is similar the proof of a.

Theorem 4.7 Let $\left(X, \tau_{1}, \tau_{2}, E\right)$ and $\left(Y, \eta_{1}, \eta_{2}, E\right)$ be two soft bitopological spaces and $f:\left(X, \tau_{1}, \tau_{2}, E\right) \rightarrow$ $\left(Y, \eta_{1}, \eta_{2}, E\right)$ be a soft mapping. Then, $f$ is soft p-open (closed) mapping iff $f:\left(X, \tau_{1}, E\right) \rightarrow\left(Y, \eta_{1}, E\right)$ and $f:\left(X, \tau_{2}, E\right) \rightarrow\left(Y, \eta_{2}, E\right)$ are soft open (closed) mappings.

Proof. It is easily obtained similar to the proof of Theorem 4.4.

Definition 4.3 Let $\left(X, \tau_{1}, \tau_{2}, E\right)$ and $\left(Y, \eta_{1}, \eta_{2}, E\right)$ be two soft bitopological spaces and $f:\left(X, \tau_{1}, \tau_{2}, E\right) \rightarrow$ $\left(Y, \eta_{1}, \eta_{2}, E\right)$ be a soft mapping. Then $f$ is called a soft pair-wise homeomorphism(soft p-homeomorphism, briefly), if

i. $f$ is a soft bijection,

ii. $f$ is a soft p-continuous,

iii. $f^{-1}$ is a soft p-continuous.

Theorem 4.8 Let $\left(X, \tau_{1}, \tau_{2}, E\right)$ and $\left(Y, \eta_{1}, \eta_{2}, E\right)$ be two soft bitopological spaces and $f:\left(X, \tau_{1}, \tau_{2}, E\right) \rightarrow$ $\left(Y, \eta_{1}, \eta_{2}, E\right)$ be a soft bijection mapping. The the following conditions are equivalent:

1. $f$ is a soft p-homeomorphism,

2. $f$ is a soft $\mathrm{p}$-continuous and soft $\mathrm{p}$-closed mapping,

3. $f$ is a soft $\mathrm{p}$-continuous and soft $\mathrm{p}$-open mapping.

A study of soft bitopological spaces is a generalization of the study of soft topological space. Therefore we intoroduce soft pair-wise neighborhood system in soft bitopological spaces and their some properties. Finally, we define the soft pair-wise continuity and soft pair-wise open (closed) mapping in soft bitopological spaces and their basic theorems are proofed. We hope that the results of this study may help in the investigation of soft pairwise seperation axioms in soft bitopological spaces.

\section{References}

[1] Aygunoğlu, A.; Aygun, H. Some notes on soft topological spaces,. Neural Computing \& Applications. 2011; 1007/s00521-011-0722-3.

[2] Babitha, K.V.; Sunil, J.J. Soft set relations and functions Computers \& Mathematics with Applications. 2010; 60:1840-1849.

[3] Bayramov, S.; Gunduz C. Soft locally compact spaces and soft paracompact spaces, Journal of Mathematics and System Science. 2013; 3, 122-130.

[4] Çağman, N.; Karatas, S.; Enginoğlu, S. Soft topology, Computers \& Mathematics with Applications. 2011; 351358.

[5] El-Sheikh, S.A.; Abd El-latif, A,M. Decompositions of some types of supra soft sets and soft continuity, International Journal of Mathematics Trends and Technology. 2014; 9(1), 37-56.

[6] Gunduz, A.C.; Sonmez, A.; Çakall, H. On soft Mappings, Proceedings of the $13^{\text {th }}$ International Conference on Computational and Mathematical Methods in Science and Engineering (CMMSE) (2013).

[7] Hussain, S.; Ahmad B. Some properties of soft topological spaces. Computers \& Mathematics with Applications. 2011; 62, 4058-4067.

[8] Ittanagi, B.M. Soft bitopological spaces, International Journal of Computer Applications. 2014; 107(7), 1-4.

[9] Kandil, A.; Tantawy, O.A.E.; El-Sheikh S. A.; Hazza S. A. Pairwise open (closed) soft sets in soft bitopological spaces, Annals of Fuzzy Math. and Infor 2016; 11(4), 571-588. 
31.

[10] Kelly, J.C. Bitopological spaces, Proc. London Math. Soc. 1963; (3)13, 71-89.

[16] Nazmul, S.K.; Samanta, S.K. Neighbourhood properties of soft topological spaces, Annals of Fuzzy Mathematics and Informatics. 2013; 6 (1), 1-15.

[11] Maji, P.K.; Roy, A.R. An Application of Soft Sets in a Decision Making Problem. Computers \& Mathematics with Applications. 2002; 44, 1077-1083.

[12] Maji, P.K.; Bismas, R.; Roy, A.R. Soft Set Theory, Computers \& Mathematics with Applications. 2003; 45, $555-562$.

[13] Mashhour, A.S.; Allam, A.A.; Mahmoud, F.S.; Khedr, F.H. On supratopological spaces, Indian J. pure appl. Math., 1983; 14(4), 502-510.

[14] Min, W.K. A note on soft topological spaces, Computers \& Mathematics with Applications. 2011; 62, 3524-3528.

[15] Molodtsov, D. Soft Set Theory-First Results, Computers \& Mathematics with Applications. 1999; 37, 19-

[17] Ozturk, T.Y.; Bayramov, S. Soft mapping spaces, The Scientific World Journal 2014; Article ID 307292, 8p.

[18] Senel, G.; Cagman, N. Soft closed sets on soft bitopological spaces, Journal of New Theory 2014; 5, 57-66.

[19] Shabir, M.; Naz, M. On soft topological spaces, Computers \& Mathematics with Applications. 2011; 61, 1786-1799.

[20] Shabir, M.; Bashir, A. Some properties of soft topological spaces. Computers \& Mathematics with Applications. 2011; 62, 4058-4067.

[21] Zorlutuna, İ.; Akdağ, M.; Min, W.K.; Atmaca, S. Remarks on soft topological spaces. Annals of Fuzzy Mathematics and Informatics. 2012; 3, 171-185. 